\title{
Vortices in Superfluid Fermi Gases through the BEC to BCS Crossover
}

\author{
Rajdeep Sensarma, Mohit Randeria, and Tin-Lun Ho \\ Department of Physics, The Ohio State University, Columbus, Ohio 43210, USA
}

(Received 27 October 2005; published 9 March 2006)

\begin{abstract}
We have analyzed a single vortex at $T=0$ in a $3 \mathrm{D}$ superfluid atomic Fermi gas across a Feshbach resonance. On the BCS side, the order parameter varies on two scales: $k_{F}^{-1}$ and the coherence length $\xi$, while only variation on the scale of $\xi$ is seen away from the BCS limit. The circulating current has a peak value $j_{\max }$ which is a nonmonotonic function of $1 / k_{F} a_{s}$ implying a maximum critical velocity $\sim v_{F}$ at unitarity. The number of fermionic bound states in the core decreases as we move from the BCS to the $\mathrm{BEC}$ regime. Remarkably, a bound state branch persists even on the $\mathrm{BEC}$ side reflecting the composite nature of bosonic molecules.
\end{abstract}

The recent discovery of vortices in ${ }^{6} \mathrm{Li}$ is a milestone in the study of superfluidity in atomic Fermi gases [1]. For the first time, the phase coherence of a superfluid atomic Fermi gas is demonstrated unambiguously. Fermi gases of alkalimetal atoms, however, are very unusual many-body systems. Their interactions, which are related to $s$-wave scattering length, are highly tunable using Feshbach resonance. By tuning the inverse scattering length $1 / a_{s}$ continuously from $-\infty$ to $+\infty$, the ground state of these systems changes from a weak coupling BCS superfluid to a molecular Bose-Einstein condensate (BEC) [2]. The region of infinite scattering (or $a_{s}^{-1}=0$ ) is particularly interesting, where the Fermi gas exhibits universal behavior, in the sense that the interaction energy scale at $T=0$ is independent of atomic details and is given by Fermi energy [3]. The physics of these Fermi gases is also related to those of strongly interacting nuclei which also have large scattering lengths. Universality implies that the superfluid transition temperature $T_{c}$ is comparable to Fermi temperature $T_{\mathrm{F}}$. In fact, $T_{c} / \epsilon_{F} \sim 0.2$ [4] at resonance, the highest of all known fermion superfluids.

Although all vortices of $s$-wave BCS superfluids have topologically invariant properties like quantized circulation of $h / 2 M$, other features like vortex core size, circulating current, and the bound state spectrum depend on dynamical details. It is natural to ask how the properties of a vortex change as one goes across the Feshbach resonance from the BCS to the BEC side, and how unitarity manifests itself in these properties. The goal of this Letter is to answer these questions using the Bogoliubovde Gennes (BdG) approach [5] at $T=0$ in a three dimensional system for a wide resonance [6]. Because of the connection between strongly interacting atomic Fermi gases with other strongly interacting Fermi systems, our results will have implications beyond cold atom physics. We find that

(A) On the BCS side, the order parameter $\Delta(\rho)$ for a single vortex exhibits two length scales: an initial rise on the scale of $k_{F}^{-1}$ [7] for which we give an elementary analytical argument, and an eventual approach to its bulk value $\Delta_{0}$ on the coherence length scale $\xi=\hbar v_{F} / \Delta_{0}$ where $v_{\mathrm{F}}$ is the Fermi velocity. At unitarity where $\Delta_{0} \sim \epsilon_{F}=$ $\hbar^{2} k_{F}^{2} / 2 M, \xi$ reduces to $k_{F}^{-1}$, and the two length scales coincide. On the BEC side the $\Delta(\rho)$ reaches its bulk value over the coherence length $\xi \sim 1 / \sqrt{n \bar{a}_{s}}$, where $n$ is the density. We also find that the density $n(\rho)$ is depleted in the vortex core, and its value $n(0)$ at the center is dramatically reduced as one goes from the BCS to the BEC limit.

(B) The circulating current $j(\rho)$ around the vortex core has a peak value $j_{\max }$ which is nonmonotonic across the resonance and reaches a maximum precisely at unitarity. Its scale is set by the critical velocity $v_{c}$ which is determined by pair breaking on the BCS side, a single-particle effect, but by collective excitations on the BEC side. $j_{\max }$ is one of the very few properties of atomic Fermi gases that varies nonmonotonically across resonance, in contrast to most thermodynamic properties.

(C) We find that unitarity represents the most robust superfluid state in the entire BCS-BEC crossover. Not only does one obtain the highest $T_{c}$ at unitarity (which is, however, not too different from the $T_{c}$ value [10] for all $1 / k_{F} a_{s}>0$ ), but one also obtains the highest critical velocity $v_{c} \sim v_{\mathrm{F}}$.

(D) We find that as we go from the BCS to the BEC limit, the number of fermionic bound states in the vortex core decreases, with a corresponding increase in both the energy of the lowest bound state and their level spacing. Remarkably, we find that a bound state is observed way past unitarity, deep into the bosonic regime, which is unique to the molecular BEC. We also find that motion along the vortex core broadens a bound state of angular momentum $\ell$ into a band with $k_{z}$ dispersion.

The BdG approach was first used to study vortices in BCS superconductors in the classic work of Caroli, de Gennes, and Matricon [5]; see also Ref. [9]. In the superfluid atomic Fermi gases, the BdG approach has been used in Ref. [11] in the BCS limit and in Ref. [12]. The latter work is in $2 \mathrm{D}$ and is implicitly restricted to "narrow resonances," which have very different physics from the wide resonances relevant to all current experi- 
ments $[6,13]$. The vortex problem has also been studied using hydrodynamic [14] and microscopic density functional [15] approaches. However the results (B), (C), and (D) mentioned above have not been reported before.

Bogoluibov-de Gennes approach. For a wide resonance, it is sufficient to use the single channel model [6]. The BdG equations in this case are

$$
\left(\begin{array}{cc}
\hat{T} & \Delta(\mathbf{r}) \\
\Delta^{*}(\mathbf{r}) & -\hat{T}^{*}
\end{array}\right)\left(\begin{array}{l}
u_{n}(\mathbf{r}) \\
v_{n}(\mathbf{r})
\end{array}\right)=E_{n}\left(\begin{array}{c}
u_{n}(\mathbf{r}) \\
v_{n}(\mathbf{r})
\end{array}\right)
$$

where $\hat{T}=-\hbar^{2} \nabla^{2} / 2 M-\mu, \mu$ is the chemical potential, $E_{n}$ the eigenvalues, and the eigenfunctions satisfy

$$
\int d^{3} \mathbf{r}\left[u_{m}^{*}(\mathbf{r}) u_{n}(\mathbf{r})+v_{m}^{*}(\mathbf{r}) v_{n}(\mathbf{r})\right]=\delta_{m n} .
$$

The order parameter $\Delta(\mathbf{r})$ and the chemical potential $\mu$ are determined by the self-consistency equation $\Delta(\mathbf{r})=$ $g \sum_{n} u_{n}(\mathbf{r}) v_{n}^{*}(\mathbf{r})$ and the average density $n=$ $2 \sum_{n} \int d^{3} \mathbf{r}\left|v_{n}(\mathbf{r})\right|^{2}$. The $\sum_{n}$ is restricted to $0 \leq E_{n} \leq E_{c}$ where $E_{c}$ is a high energy cutoff (discussed below). From now on we measure all energies in units of $\epsilon_{F}$ and lengths in units of $k_{F}^{-1}$, so that the bare attraction $g$ has units of $\epsilon_{F} / k_{F}^{3}$. The cutoff $E_{c}$ and the corresponding $g\left(E_{c}\right)$ should be chosen such that $[2,10]$

$$
\frac{1}{k_{F} a_{s}}=-\frac{8 \pi \epsilon_{F}}{g k_{F}^{3}}+\frac{2}{\pi} \sqrt{\frac{E_{c}}{\epsilon_{F}}}
$$

so that the low energy effective interaction is described by the scattering length $a_{s}$.

We work in cylindrical coordinates $\mathbf{r}=(\rho, \theta, z)$ in a gauge in which $\Delta(\mathbf{r})=\Delta(\rho) e^{-i \theta}$. Working in a cylindrical box of radius $R$ and height $L$, our normalized wave functions are of the form $u_{n}(\mathbf{r})=u_{n}(\rho) e^{i \ell \theta} e^{i k_{z} z} / \sqrt{2 \pi L}$ and $v_{n}(\mathbf{r})=v_{n}(\rho) e^{i(\ell+1) \theta} e^{i k_{z} z} / \sqrt{2 \pi L}$ so that (1) decouples into different $l$ and $k_{z}$ sectors [9]. We further expand the radial wave functions $u_{n}(\rho)=\sum_{j} c_{n j} \phi_{j \ell}(\rho)$ and $v_{n}(\rho)=$ $\sum_{j} d_{n j} \phi_{j \ell+1}(\rho)$ in the orthonormal basis set $\phi_{j \ell}(\rho)=$ $\sqrt{2} J_{\ell}\left(\alpha_{j \ell} \rho / R\right) /\left[R J_{\ell+1}\left(\alpha_{j \ell}\right)\right]$ where $\alpha_{j \ell}$ is the $j$ th zero of $J_{\ell}(x)$. The BdG eigenvalue equation now reduces to a matrix diagonalization problem

$$
\left(\begin{array}{cc}
T_{\ell} & \Delta_{\ell} \\
\Delta_{\ell+1} & -T_{\ell+1}
\end{array}\right) j\left(\begin{array}{l}
c_{n j^{\prime}} \\
d_{n j^{\prime}}
\end{array}\right)=E_{n}\left(\begin{array}{l}
c_{n j} \\
d_{n j}
\end{array}\right)
$$

where $\quad T_{\ell}^{j j^{\prime}}=\left(\alpha_{j \ell}^{2} / R^{2}+k_{z}^{2}-\mu\right) \delta_{j j^{\prime}} \quad$ and $\quad \Delta_{\ell}^{j j^{\prime}}=$ $\int d \rho \Delta(\rho) \phi_{j \ell}(\rho) \phi_{j^{\prime} \ell+1}(\rho)$. While the different $\ell$ and $k_{z}$ sectors are completely decoupled in (4), they are coupled through the self-consistency equations [16]. Since the BdG equations are invariant under $E_{n} \rightarrow-E_{n}, u_{n}(\mathbf{r}) \rightarrow v_{n}^{*}(\mathbf{r})$, $v_{n}(\mathbf{r}) \rightarrow-u_{n}^{*}(\mathbf{r})$, one can simply get the positive energy eigenfunctions for negative $\ell$ by looking at the negative energy eigenfunctions for positive $\ell$.

Ideally one would like to take $E_{c} \rightarrow \infty$ and obtain solutions independent of this cutoff. In practice, the size of Hilbert space grows like $R L \sqrt{E_{c}}$ and to make the calculation manageable we choose $E_{c}=9 \epsilon_{F}, \quad R=$ $25 k_{F}^{-1}$, and $L=10 k_{F}^{-1}$. We have checked that for this choice of cutoff, (larger than that in Ref. [12]), our results in the uniform case are no more than 5\% different from the infinite cutoff answers at unitarity.

Vortex core structure. The order parameter $\Delta(\rho)$ is plotted in Fig. 1 for various values of $1 / k_{F} a_{s}$. The weak oscillations in $\Delta(\rho)$, most prominent in the BCS regime, are likely finite size effects with no physical significance [17]. Another very interesting aspect of the BCS regime, clearly visible in Fig. 1, is the presence of two length scales $k_{F}^{-1}$ and $\xi$ in the $T=0$ result for $\Delta(\rho)$. This is in marked contrast to the Ginzburg-Landau result where $\Delta_{\mathrm{GL}}(\rho) \sim$ $\tanh (\rho / \xi)$. While this effect was, in fact, recognized in the early superconductivity literature [8] in an Eilenberger calculation, we provide an elementary analytical derivation here. Close to the origin, one may ignore $\Delta$ relative to the kinetic energy terms in the BdG equations and find $u_{\ell}=$ $A_{\ell}^{-1} J_{\ell}\left(k_{F} \rho\right), v_{\ell}=A_{\ell}^{-1} J_{\ell+1}\left(k_{F} \rho\right)$ where $A_{\ell}$ are constants. For small $\rho$ we thus find $\Delta(\rho)=g u_{0}(\rho) v_{0}(\rho)=A_{0}^{-2} k_{F} \rho$. We next determine $A_{0}$ as follows. For large $\rho, \Delta(\rho)=\Delta_{0}$ and $u_{0} \sim\left(1 / \sqrt{B_{0} \rho}\right) \cos \left(k_{F} \rho\right) \exp (-\rho / \xi)$ and $v_{0}=$ $\left(1 / \sqrt{B_{0} \rho}\right) \sin \left(k_{F} \rho\right) \exp (-\rho / \xi)$ (up to irrelevant phase shifts) where $B_{0}$ is another constant. Matching the large and small $\rho$ solutions at $\rho=\xi$, we get $A_{0} \sim\left(B_{0} k_{F}^{-1}\right)^{1 / 2}$ [18]. Finally, using the normalization condition (2) we can fix the constant $A_{0}=\xi /\left(k_{F} \xi\right)^{1 / 2}$ which leads to the result $\Delta(\rho) \sim \Delta_{0} k_{F} \rho$ for small $\rho$. Thus in the BCS limit, the initial slope of $\Delta(\rho)$ is set by $k_{F}^{-1}$ although the eventual approach to its uniform value $\Delta_{0}$ is on a second scale of $\xi=\hbar v_{F} / \Delta_{0} \gg k_{F}^{-1}$.

We see that outside of the BCS regime there appears to be a single length scale in $\Delta(\rho)$ as seen in Fig. 1. As the coupling increases toward unitarity the order parameter value at large $\rho, \Delta_{0}$ increases toward $\epsilon_{F}$ and the scale $\xi$ shrinks to $\sim k_{F}^{-1}$. The overall behavior of $\Delta(\rho)$ across the resonance is shown in Fig. 2(a). At the same time the density profile $n(\rho)$ around the vortex evolves as shown

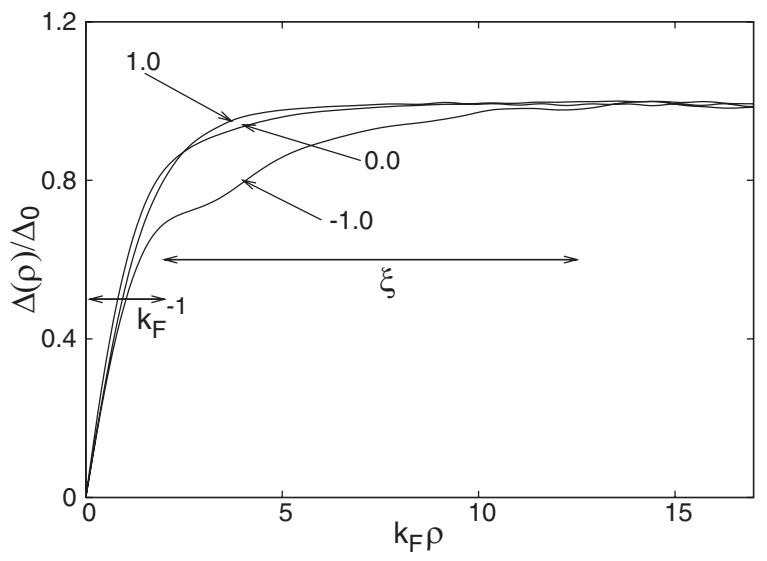

FIG. 1. The order parameter normalized by its value far from the vortex for three different couplings $1 / k_{F} a_{s}=-1,0,1$. Note the two length scales in the BCS limit $1 / k_{F} a_{s}=-1$. 
in Fig. 2(b). Near the center of the vortex $n(\rho) \simeq n(0)+$ $a \rho^{2}$. The density at the center $n(0)$ is a strongly decreasing function of $1 / k_{F} a_{s}$ as shown in the inset of Fig. 2(b) dropping from approximately $0.8 n$ at $1 / k_{F} a_{s}=-1$, corresponding to a nearly "full" core, to $0.1 n$ at $1 / k_{F} a_{s}=$ +1 , which is a nearly "empty" core.

Circulating current. The current circulating around the vortex core is $\mathbf{j}=\rho_{s} \mathbf{v}_{s}$, where $\rho_{s}$ is the local superfluid density and $\mathbf{v}_{s}=(\hbar / 2 M \rho) \hat{\theta}$. Far away from the vortex core, $j \sim 1 / \rho$ since $\rho_{s}$ is constant. On the other hand, near the vortex core, $\rho_{s} \sim \Delta^{2}$, so that $j \sim \rho$. With decreasing $\rho$, the current initially increases until it reaches a maximum, $j_{\max }$, at $\rho^{*}$. For $\rho<\rho^{*}$ the kinetic energy cost of current flow is much larger than the condensation energy and the superfluid order parameter is suppressed at the center of the vortex. In this sense, the maximum current density at $\rho^{*}$ gives us an estimate of the critical current beyond which superfluid order is destroyed.

Formally the current density is given by

$$
\mathbf{j}(\rho)=-\frac{2 \hbar}{2 \pi L m \rho} \sum_{n l k_{z}}(l+1)\left[\sum_{j} d_{n j} \phi_{j l+1}(\rho)\right]^{2} \hat{\boldsymbol{\theta}} .
$$

The profiles of $j(\rho)$ for various $1 / k_{F} a_{s}$ are shown in Fig. 3(a). They all show a peak, as explained above. We find that [19] the location $\rho^{*}$ of the maximum current shows a weak nonmonotonic behavior in the vicinity of unitarity, which is roughly consistent with the nonmonotonic behavior of the coherence length $\xi$ as a function of $1 / k_{F} a_{s}$ predicted earlier; see Fig. 3 of Ref. [20].

The peak current $j_{\max }$, however, shows a nonmonotonic behavior as a function of $1 / k_{F} a_{s}$, peaking at unitarity as shown in Fig. 3(b). We may understand this nonmonotonic behavior as follows. On the BCS side, the critical velocity, determined by depairing, is $\Delta_{0} / k_{F}$ and thus $j_{\max } \sim$ $n v_{\mathrm{F}}\left(\Delta_{0} / \epsilon_{F}\right)$. The critical current thus increases as one moves toward unitarity from the BCS side as $\Delta_{0}$ increases. At unitarity, universality dictates that the critical velocity
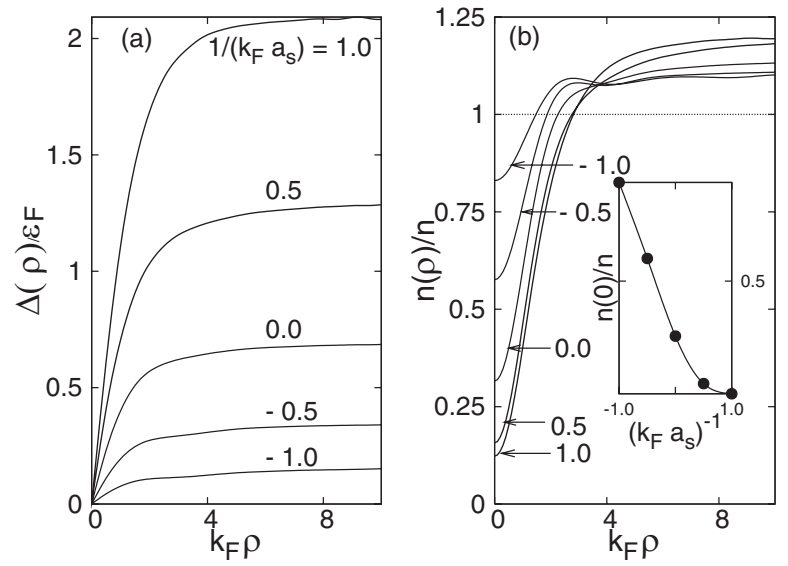

FIG. 2. (a) The order parameter profile and (b) the density profile for different values of $1 /\left(k_{F} a_{s}\right)$. Inset: the density at the center of vortex as function of $1 /\left(k_{F} a_{s}\right)$. must be of order $v_{\mathrm{F}}$ so that $j_{\max } \sim n v_{\mathrm{F}}$. As one goes toward the BEC side the critical current is now determined not by pair breaking but rather by the collective excitations. The Landau criterion suggests $v_{c} \sim v_{\mathrm{F}} \sqrt{k_{F} a_{s}}$ which leads to a $j_{\max }$ which decreases with increasing $1 / k_{F} a_{s}$. We thus find the interesting result that the mechanism for destruction of superfluidity, as reflected in the maximum current $j_{\max }$, changes as one goes across the resonance from pair breaking on the BCS side to collective on the BEC side.

Spectrum. We now turn to the fermionic bound states in the vortex core and their evolution through the BCSBEC crossover. The BCS-limit results are very well-known [5]. Caroli, de Gennes, and Matricon [5] showed that for each $\ell$ and $k_{z}$ there is a bound state, with energy less than $\Delta_{0}$, in the core of the vortex. The lowest energy fermionic excitation (with $\ell=0$ and $k_{z}=0$ ) has a "minigap" $\Delta_{0}^{2} / 2 \epsilon_{F} \ll \Delta_{0}$. As we increase $1 / k_{F} a_{s}$ the spectrum changes as shown in Figs. 4(a) and 4(b). For clarity we show the spectrum only as a function of angular momentum $\ell$ at fixed $k_{z}=0$ ( $k_{z}$ dispersion is discussed later).

As long as the chemical potential $\mu>0$ (which includes unitarity), the bound (continuum) states are those with energies smaller (larger) than $\Delta_{0}$. The bound state spectrum at unitarity [Fig. 4(a)] is not qualitatively different from the BCS limit, except that both the minigap and the level spacing are larger, and therefore one has fewer bound states. Remarkably, the minigap continues to follow $\Delta_{0}^{2} / 2 \epsilon_{F}$ even through unitarity as shown in Fig. 4(c).

Once the chemical potential $\mu<0$, as in the BEC regime shown in Fig. 4(b), the continuum of fermionic excitations exists for $E \geq\left(|\mu|^{2}+\Delta_{0}^{2}\right)^{1 / 2}$ [2]. We can still define a fermionic bound state by demanding that the corresponding wave function decays exponentially to zero away from the vortex core. If such states exist, then it can be easily shown that their energy must lie in the interval $|\mu| \leq E<\left(|\mu|^{2}+\Delta_{0}^{2}\right)^{1 / 2}$. Remarkably, we find such a bound state well into the BEC regime as evident from Fig. 4(b). It is interesting that the off-diagonal potential $\Delta(\rho)$ produces an Andreev bound state even at an
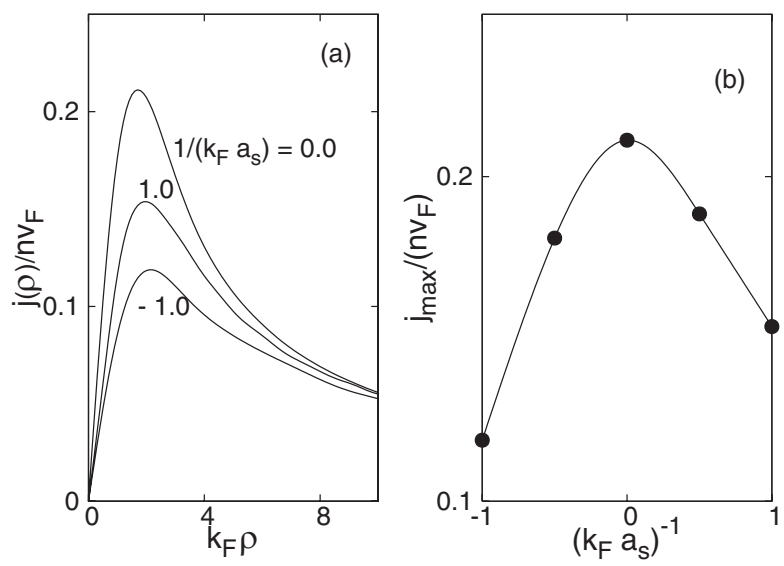

FIG. 3. (a) The current distribution for three different values of $1 /\left(k_{F} a_{s}\right)$ (b) The peak current $j_{\max }$ vs $1 /\left(k_{F} a_{s}\right)$. 

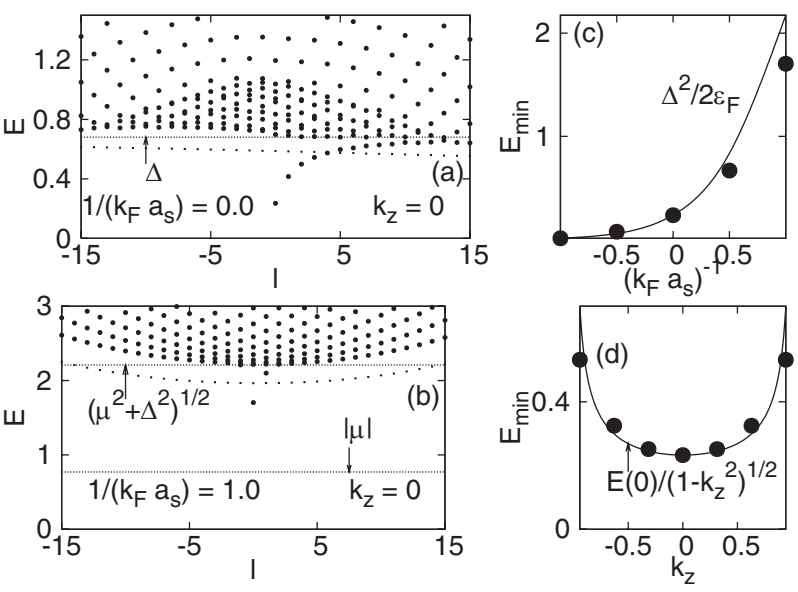

FIG. 4. The BdG spectrum in the $k_{z}=0$ sector as a function of $\ell$ for (a) $1 /\left(k_{F} a_{s}\right)=0.0$, i.e., unitarity and (b) $1 /\left(k_{F} a_{s}\right)=1.0$, i.e., on BEC side. (The smaller dots correspond to bound states living on the boundary) (c) The minigap $\left(\ell=0, k_{z}=0\right)$ as a function of $1 /\left(k_{F} a_{s}\right)$. (d) The $k_{z}$ dependence of the bound state energy for $\ell=0$ at unitarity.

energy larger than the maximum value of the potential $\Delta_{0}$, but we must emphasize that this bound state is below the continuum which starts at $\left(|\mu|^{2}+\Delta_{0}^{2}\right)^{1 / 2}$. The fermionic bound states are a unique consequence of composite nature of the bosons and are absent in atomic BEC.

As a result of motion along the vortex axis, each state for fixed angular momentum $\ell$ shown in Figs. 4(a) and 4(b) actually broadens into an energy band with $k_{z}$ dispersion. Our calculated $k_{z}$ dependence of the bound state energy for the $l=0$ bound state at unitarity is shown in Fig. 4(d). The energies (which are discrete due to the finite $L$ along $z$ ) continue to follow the BCS-limit prediction [5] $E_{0} /(1-$ $\left.k_{z}^{2}\right)^{1 / 2}$ even at unitarity.

Finally, we note that there is a branch of bound states [shown with smaller dots in Figs. 4(a) and 4(b)] which lies below the fermionic energy gap in the bulk but differs from the vortex core states (which exist only for $\ell \geq 0$ ) in that it exists both for positive and negative $\ell$. From their wave functions [19] we deduce that these states are not related to the vortex core, but are, in fact, trapped near $\rho=R$ due to the suppression of the order parameter by the hard wall boundary condition [21].

Conclusions. The physics of a vortices in the BEC to BCS crossover has led to interesting results (A) through (D) summarized in the introduction. The study of the order parameter profile in the vortex core and the circulating current may require new experimental methods which will allow more detailed diagnostics. The existence of fermionic bound states should be detectable by spectro- scopic means, or through the damping of sound, or through effects of dissipation in vortex dynamics. We hope that the unique properties of vortices that we point out here will stimulate further experimental and theoretical studies on new methods to probe strongly interacting Fermi gases across Feshbach resonance.

This work is supported by NASA Grant No. NAG8-1765 and NSF Grant No. DMR-0426149.

[1] M. W. Zwierlein et al., Nature (London) 435, 1047 (2005).

[2] A. J. Leggett in Modern Trends in the Theory of Condensed Matter, edited by A. Pekalski and R. Przystawa (Springer-Verlag, Berlin, 1980); M. Randeria in Bose Einstein Condensation, edited by A. Griffin, D. Snoke, and S. Stringari (Cambridge, Cambridge, England, 1995).

[3] See K. O'Hara et al., Science 298, 2179 (2002); T. L. Ho, Phys. Rev. Lett. 92, 090402 (2004), and references therein.

[4] C. A. Regal, M. Greiner, and D. S. Jin, Phys. Rev. Lett. 92, 040403 (2004); M. W. Zwierlein et al., Phys. Rev. Lett. 92, 120403 (2004).

[5] C. Caroli, P. de Gennes, and J. Matricon, Phys. Lett. 9, 307 (1964).

[6] That current experiments are for wide resonances is emphasized in R. Diener and T. L. Ho, cond-mat/0405174; See also D. S. Petrov et al., cond-mat/0502010.

[7] While it was recognized in earlier work [8,9], this fact nevertheless does not seem to be widely appreciated.

[8] L. Kramer and W. Pesch, Z. Phys. 269, 59 (1974).

[9] F. Gygi and M. Schlüter, Phys. Rev. B 43, 7609 (1991); J. D. Shore et al., Phys. Rev. Lett. 62, 3089 (1989).

[10] C. A. R. Sá de Melo, M. Randeria, and J. R. Engelbrecht, Phys. Rev. Lett. 71, 3202 (1993).

[11] N. Nygaard et al., Phys. Rev. Lett. 90, 210402 (2003).

[12] M. Machida and T. Koyama, Phys. Rev. Lett. 94, 140401 (2005).

[13] A. Bulgac, cond-mat/0505524.

[14] G. Tonini and Y. Castin, cond-mat/0504612.

[15] A. Bulgac and Y. Yu, Phys. Rev. Lett. 91, 190404 (2003).

[16] $\Delta(\rho)=(g / 2 \pi L) \sum_{n j j^{\prime} l k_{z}} c_{n j} d_{n j^{\prime}} \phi_{j l}(\rho) \phi_{j^{\prime} l+1}(\rho)$ and $n=$ $\left(2 / \pi R^{2} L\right) \sum_{n j l k_{z}} d_{n j}^{2}$ are the self-consistency equations.

[17] We believe that the smooth $\Delta$ profile seen in Ref. [9], which used a BCS interaction and a very large system size, shows that the oscillations seen in the BCS limit of our dilute gas model are a finite size artifact, and not Friedellike oscillations as suggested in Refs. [11,12].

[18] Equating $A_{0}^{-1} J_{0}\left(k_{F} \xi\right)=\left(B_{0} \xi\right)^{-1 / 2} \cos \left(k_{F} \xi\right) e^{-1}$, and using $J_{0}(x) \sim \cos x / \sqrt{x}$ for $x \gg 1$, we have $A_{0} \sim\left(B_{0} k_{F}^{-1}\right)^{1 / 2}$.

[19] R. Sensarma, M. Randeria, and T. L. Ho (unpublished).

[20] J. R. Engelbrecht, M. Randeria, and C. A. R. Sa de Melo, Phys. Rev. B 55, 15153 (1997).

[21] Y. Ohashi and A. Griffin, Phys. Rev. A 72, 013601 (2005). 